\title{
Quality of Chest Compressions on A Dental Chair -- A Randomized Simulation Trial as Observation in Support of A Procedure Proposal
}

\author{
Tomasz Kłosiewicz ${ }^{1, \star}$, Mateusz Puślecki ${ }^{1,2}$, Radosław Zalewski ${ }^{1}$, Michał Mandecki ${ }^{1}$, \\ Ilona Skitek-Adamczak ${ }^{1}$, Maciej Sip ${ }^{1}$, Marek Dąbrowski ${ }^{3}$, Martyna Ratajczak ${ }^{4}$, \\ Przemysław Rachubiński ${ }^{5}$, Bogusz Szczepański ${ }^{5}$, Marek Dorożyński ${ }^{6}$, \\ Beata Czarnecka7 ${ }^{7}$, Bartłomiej Perek ${ }^{2}$
}

\author{
${ }^{1}$ Department of Medical Rescue, Poznan \\ University of Medical Sciences, Poznan, \\ Poland \\ ${ }^{2}$ Department of Cardiac Surgery and \\ Transplantology, Poznan University of \\ Medical Sciences, Poznan, Poland \\ ${ }^{3}$ Chair and Department of Medical \\ Education, Poznan University of Medical \\ Sciences, Poznan, Poland \\ ${ }^{4}$ Department of Emergency Medicine, \\ Poznan University of Medical Sciences, \\ Poznan, Poland \\ ${ }^{5}$ Faculty of Health Sciences, Poznan \\ University of Medical Sciences, Poznan, \\ Poland \\ ${ }^{6}$ Medical Faculty, Poznan University of \\ Medical Sciences, Poznań, Poland \\ ${ }^{7}$ Chair and Department of Biomaterials \\ and Experimental Dentistry, Poznań, \\ Poland \\ *Correspondence \\ klosiewicz.tomek@gmail.com \\ (Tomasz Kłosiewicz)
}

\begin{abstract}
Background: Although medical emergencies among dental patients are not frequent, several factors may provoke sudden cardiac arrests. Early initiation of high-quality chest compressions (CC) is of crucial importance for the safety and effectiveness of cardiopulmonary resuscitation (CPR). Aims: We aimed to evaluate the quality of chest compressions performed on a dental chair for the proposed procedure in case of cardiac arrest in a dental office. Methods: We designed a prospective, randomized, crossover simulation study. Sixty paramedic students were randomly assigned to the control group, in which resuscitation was performed on the floor $(\mathrm{n}=30)$ or to the experimental group, in which compressions were performed on a dental unit $(n=30)$. We used a simulator that recorded number of compressions, rate, depth of compressions and chest recoil. Results: There were no significant differences in numbers, rate, depth of chest compressions or in chest recoil between groups. Conclusions: We proved that performing chest compressions on a dental chair might be as effective as on the floor. On this basis, we propose a procedure for safe and efficient performance of CPR in a dental office.
\end{abstract}

\section{Keywords}

Chest compressions, Dental equipment, Patient safety, Resuscitation, Simulation training

\section{Introduction}

Life-threatening events in a dental office are not frequent. Among them, approximately $30 \%$ of cases are regarded as vasovagal syncopes followed by convulsions, choking on a foreign body, hypertensive crisis, asthma attacks, chest pain, metabolic disorders (e.g. hypoglycaemia), anaphylactic reactions and hyperventilation [1-3]. According to medical data, up to $40 \%$ of cases require emergent medical interventions [4]. The incidence of emergencies at British academic dental clinics is estimated at 1.8 cases per year [5]. It has been calculated that during the $20-30$ years of professional practice, each dentist may witness from 5 to 7 life-threatening situations [6]. Although the majority of them affects patients undergoing treatment, $10 \%$ to even more than $30 \%$ may refer to nonpatients [7]. German authors mentioned that the number of medical emergency cases in a dental office amounts to several cases per year [8].

Apart from chronic conditions, some of medical procedures performed in a dental office may predispose or even lead to life-threatening situations. Risk factors include airway obstruction due to tissue oedema, aspiration of saliva, blood, rinsing agents or other materials and tools. Moreover, local anaesthetics, antibiotics, disinfectants and latex also pose a risk of anaphylactic reactions [9]. According to published reports, the incidence of sudden cardiac arrest (SCA) in dental surgeries is from 0.3 to $1 \%$. It constitutes to $0.002-0.011$ cases per dentist per year $[10,11]$. Only $41 \%$ of the dentists judged themselves capable of diagnosing the cause of life-threatening events during a dental visit [3].

When alarming symptoms occur, immediate implementation of appropriate procedures is crucial for patient's chances of survival. The above-described life-threatening conditions of dental patients may lead to SCA before emergency medical services arrive. Thus, medical staff in a dental office should be theoretically and practically prepared for such interventions. There is still no clear procedure that could be used by inexperienced dental professionals. There are studies presenting the quality of $\mathrm{CC}$ in a dental chair. However, the results refer to already outdated recommendations [12]. Based on our professional experience in both emergency rescue teams and simulation-based education, we have decided to develop a procedure of CPR as a treatment of SCA cases in a dental 


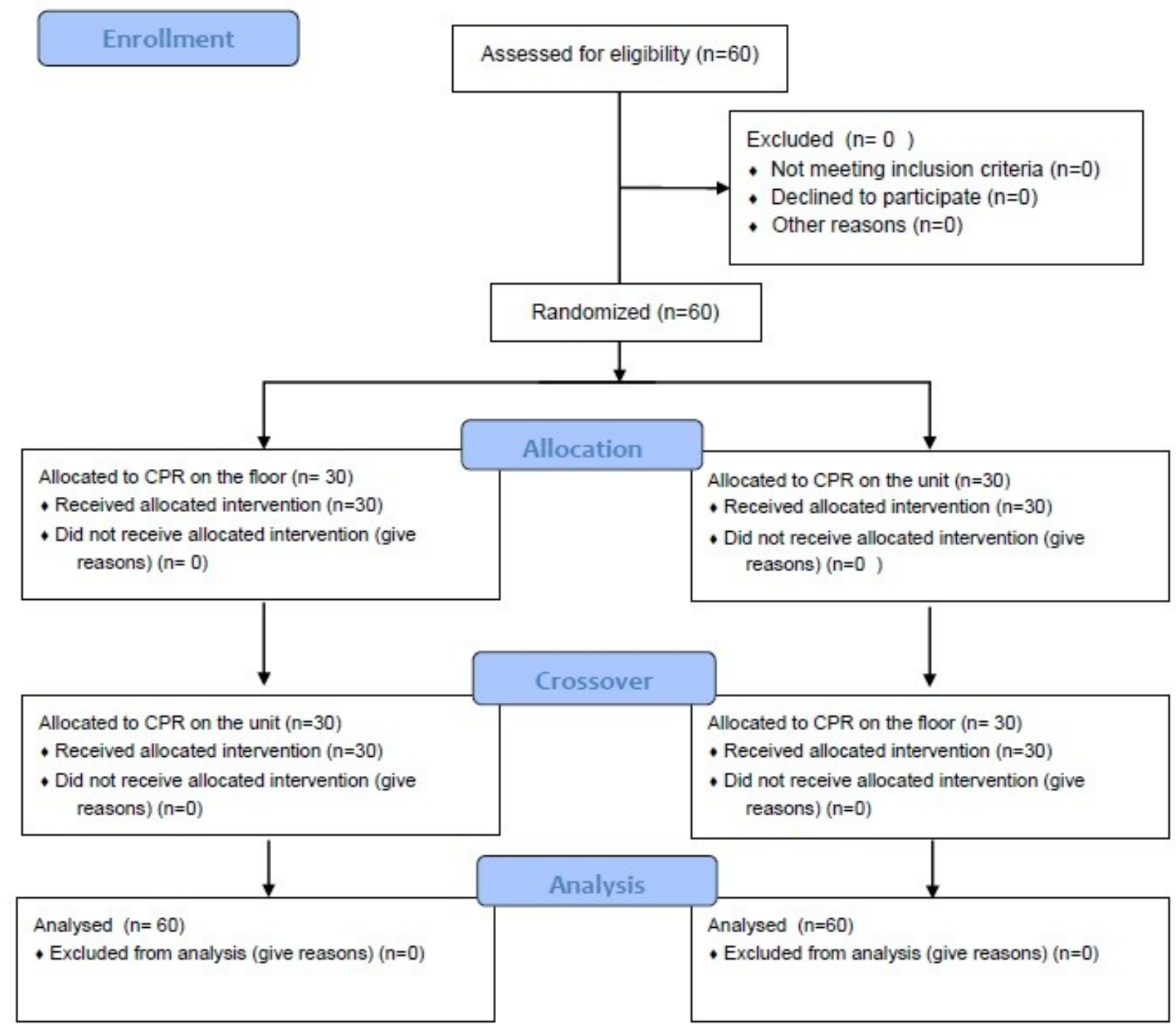

F I G U R E 1. The study protocol based on CONSORT 2010 Statement.

office. We aimed to evaluate the quality of $\mathrm{CC}$ performed on a dental unit for the proposed procedure in case of SCA in a dental office.

\section{Materials and methods}

\subsection{Study design and participants}

We designed a prospective, randomized, crossover simulation study. Sixty first-year paramedic students took part in the research. Students were recruited on a voluntary basis. Each student had to attend Basic Life Support (BLS) and Advanced Life Support courses prior to the study. The classes were obligatory for every student and described in the curriculum. No external funding for the courses was required. The classes concluded with a practical exam confirming CC skills. The period between the practical exam and the research lasted three months. Both classes and the research were conducted in the training rooms of the Medical Simulation Centre, with which the students were well acquainted. During the BLS and ALS training, students became acquainted with a medical simulation of low and high fidelity. However, the participants have never conducted simulated resuscitation on a dental unit before and this was a new experience for them. The manikins used in the research were the same with which the students worked during obligatory classes.

The experiment was conducted in a closed room without any additional stimuli, so that participants could focus on the task as much as possible. The only persons present in the room were the investigators. Each participant worked individually. No additional features were used in the test, such as moulage, media or props. The manikin was unable to generate any variables such as breath or pulse. No scenario was used in the test. When the participants performed two attempts (one in the control and one in the experimental group), the researchers discussed the results with each of them after both sessions.

In the pilot study 10 trials were conducted. Resuscitation was performed by paramedic students at the end of obligatory classes. The study showed that the difference in quality parameters of resuscitation performed on the floor and in a dental chair was $15 \%$. Assuming a type I error rate of $5 \%$ and power of $80 \%, 54$ medical student recruits were required for participation. 


\subsection{Intervention}

The allocation to the first group was randomized. The randomization procedure was prepared with a use of a free online randomization tool (www.randomizer.org).

In the control group, participants performed $\mathrm{CC}$ on the floor, whereas the experimental group students performed $\mathrm{CC}$ on a dental chair. $\mathrm{CC}$ were carried out for 2 minutes without interruptions, and then the groups switched, so that each student could participate in both arms of study, the control and the experimental one. Between each station, students had at least a 10-minute break. The study protocol based on CONSORT 2010 Statement was presented on Fig. 1. The KaVo Primus 1058 S/TM/C/G dental unit (Kaltenbach \& Voigt $\mathrm{GmbH}$, Biberach, Germany) was used in the study. The chair was positioned horizontally at the height of $45 \mathrm{~cm}$ above the ground. The headrests of the seat were placed as horizontally as possible and were supported by a dental stool for more excellent stability. The unit and stool used in the study as well as position of the rescuer and chair are presented in Fig. 2.

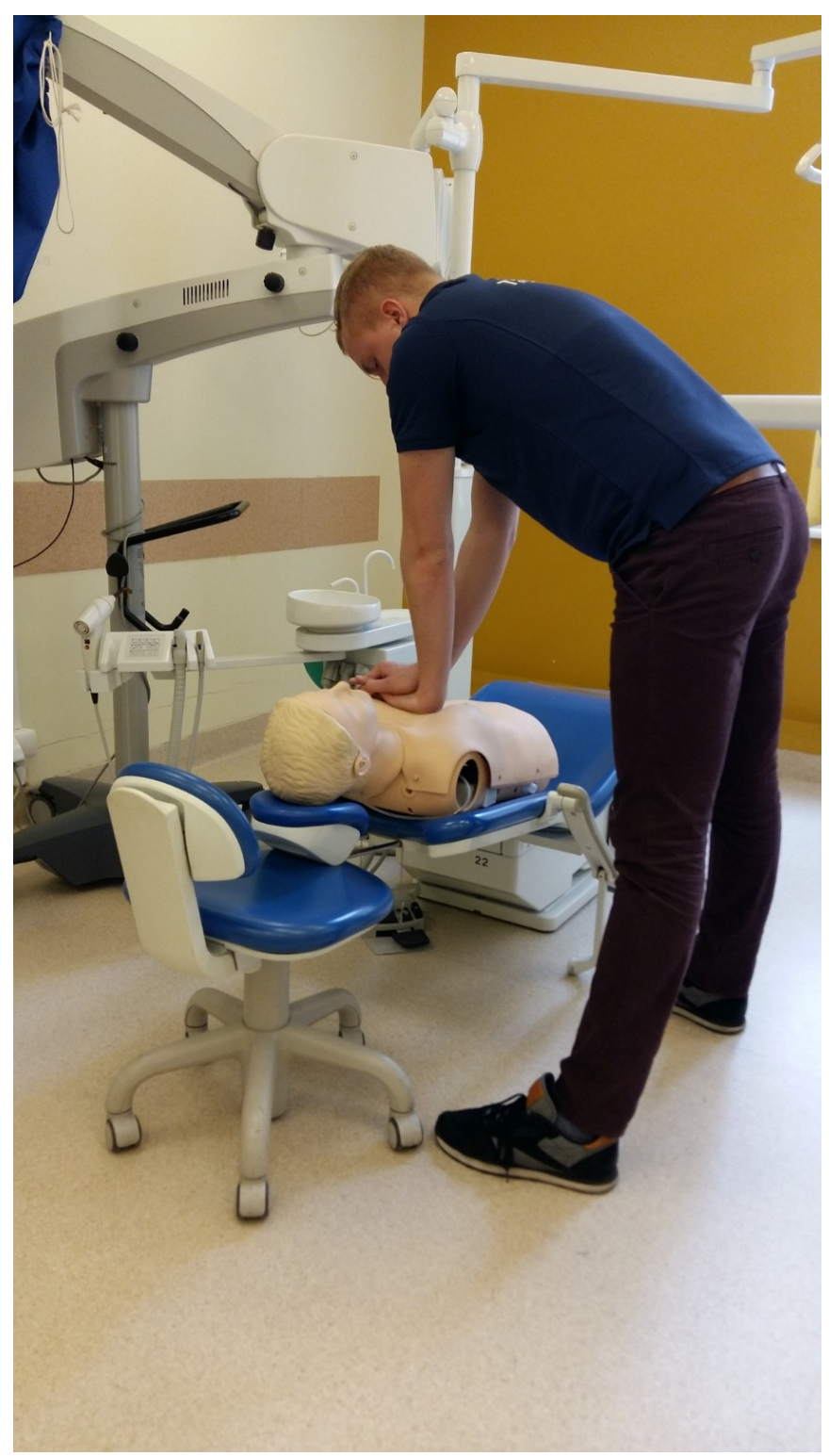

F IGURE 2. The standpoint for experimental test and position of a rescuer.

\subsection{Equipment and Outcomes}

For the assessment of parameters of CC quality, ResusciAnne ${ }^{\circledR}$ QCPR manikin (Laerdal Medical AS, Stavanger, Norway) equipped with ResusciAnne ${ }^{\circledR}$ Wireless SkillReporter ${ }^{\mathrm{TM}}$ Software was used. Each resuscitation was saved as a separate record. All the data was collected and stored in Microsoft Excel 2016. The following parameters were evaluated: 1) compression depth, 2) percent of compressions with correct depth, 3) percent of full chest recoil, 4) compression rate, 5) percent of compressions with correct rate, 6) number of compressions. Our primary outcomes were: depth and rate of $\mathrm{CC}$ and percent of full recoil. Our secondary outcomes were: number of compressions, percent of compressions with correct rate and percent of compressions with correct depth.

According to the guidelines of the European Resuscitation Council, the following quality criteria for $\mathrm{CC}$ were defined as appropriate: rate between $100-120 / \mathrm{min}$, depth between 50 $60 \mathrm{~mm}$, full chest recoil after each compression.

\subsection{Statistical analysis}

First, the quantitative variables were checked for normality with the use of the Shapiro-Wilk W test. If they satisfied normal distribution criteria, they were expressed as the means \pm standard deviation (SD). Data was presented as medians and interquartile range [IQR] if it did not satisfy normal distribution criteria. The categorical variables were expressed as the percentages (\%) with numbers (n). For statistical analysis, chi square test and t-student's were used as appropriate. A p-value of less than 0.05 was considered significant. The analysis was performed using the Statistica 13.1 software (Tibco Software Inc., Tulsa, OK, USA).

\section{Results}

Among participants, there were 33 men and 27 women. The total number of measurements was 120, 60 in each group. The mean number of compressions per one rescuer were similar, $226 \pm 17$ in the control and $224 \pm 35$ in the experimental group. This difference was not statistically significant $(\mathrm{p}=$ $0.72)$.

Median depth in the control group was $59 \mathrm{~mm}$ $[50,51,52,53,54,55,56,57,58,59,60,61,62]$, whereas in the experimental group $57 \mathrm{~mm}[48,49,50,51,52,53,54,55,56,57,58,59$, $60,61]$. Mean rate was $112 \pm 8 / \mathrm{min}$ in the control group and $111 \pm 7 / \mathrm{min}$ in the experimental group. For both parameters the differences were not statistically significant $(\mathrm{p}=0.28$ and $\mathrm{p}=0.64$ respectively)

Although a significant difference in chest compressions rate was not found, taking into consideration the incorrect rate, there were more compressions performed too fast rather than too slow in both groups. Regarding the depth of compressions, we observed a wide spread of data. In both groups there were more compressions with an incorrect depth. In this case, however, the differences in the distribution between the two groups were not statistically significant.

The detailed distribution of results is presented in Table 1 . 
TA B L E 1. Percentage of compressions in particular depth, rate and recoil ranges.

\begin{tabular}{|c|c|c|c|}
\hline \multirow[t]{2}{*}{ Variable } & \multicolumn{3}{|c|}{ Place of compressions } \\
\hline & On the floor & On the chair & p-value \\
\hline \multicolumn{4}{|c|}{ Depth of compressions [mm] } \\
\hline $50-60$ & $43.3 \%(n=26)$ & $35.0 \%(\mathrm{n}=21)$ & 0.33 \\
\hline$<50$ & $20.1 \%(n=12)$ & $31.6 \%(n=19)$ & 0.33 \\
\hline$>60$ & $36.6 \%(n=22)$ & $33.4 \%(n=20)$ & 0.33 \\
\hline \multicolumn{4}{|c|}{ Rate rate of compressions $(\min -1)$} \\
\hline $100-120$ & $80.0 \%(n=48)$ & $76.7 \%(n=46)$ & 0.86 \\
\hline$>120$ & $16.6 \%(n=10)$ & $18.3 \%(\mathrm{n}=11)$ & 0.86 \\
\hline$<100$ & $3.4 \%(n=2)$ & $5.0 \%(\mathrm{n}=3)$ & 0.86 \\
\hline \multicolumn{4}{|l|}{ Recoil (\%) } \\
\hline$>80$ & $56.7 \%(n=34)$ & $60.0 \%(n=36)$ & 0.92 \\
\hline $60-80$ & $11.7 \%(n=7)$ & $11.7 \%(\mathrm{n}=7)$ & 0.92 \\
\hline$<60$ & $31.6 \%(n=19)$ & $28.3 \%(n=17)$ & 0.92 \\
\hline
\end{tabular}

\section{Discussion}

Few factors determine high-quality of resuscitation, namely: depth of CC from $5 \mathrm{~cm}$ to $6 \mathrm{~cm}$ and an optimal rate of 100 - 120 compressions per minute, chest recoil and very short interruptions in between compressions. These parameters only within the mentioned ranges were found to provide adequate coronary perfusion pressure and increased chances to achieve the return of spontaneous circulation [13].

SCA in a dental office is rare. Nevertheless, the guidelines of the European Resuscitation Council (ERC) recommend regular training of dental personnel and increased availability of automated external defibrillator devices [14].

The primary ground on which CPR is performed in a dental office is a dental chair. Due to its design and setting, it enables immediate placement of a patient in a horizontal position, and at the same time provides a stable ground needed for effective CC. SCA patients suffer from increased muscle tension in the initial phase, which is often confused with seizures. Only after a while, the body becomes flaccid. For inexperienced persons, it may be challenging to move a patient safely from a dental unit to the floor. The improper attempt to change the body position of an unconscious patient carries the risk of injury both for a patient and for rescuers themselves [15].

It is unclear whether the time of changing a position of a unit is shorter or longer than the time, during which a patient is moved to the floor. To a large extent, it depends on the staff number and expertise. Therefore, it is the most effective to leave a patient on a unit and to start CPR immediately. Lepere et al. noted that adequate resuscitation is possible on a dental unit. They proved that it is achievable to reach CC depth of 41-50 mm [16]. Takeshi et al. have indicated that the average depth of CC carried out on a dental unit amounts to $34.8 \mathrm{~mm}$ whereas on the floor to $36.6 \mathrm{~mm}$. At the same time, the percentage of CC performed correctly was $37.8 \%$ and $49.7 \%$, respectively [17]. Current ERC guidelines are more demanding than the previous ones. The recommended
CC depth was estimated to at least $50 \mathrm{~mm}$ [13]. Our research has confirmed that such depth is manageable. Some of the compressions performed by the participants were suboptimal. There is a correlation between the rate of compressions and their depth. Excessive compression rate was found to be a major cause of inadequate CPR performance [18]. Therefore ERC recommends using real time feedback devices [13]. These devices continuously present rate and depth as well as recoil. This allows the rescuer to correct the quality of the compressions. A device which monitors the quality of $\mathrm{CPR}$ by analyzing $\mathrm{CC}$ with a force and deflection sensor may overestimate compression depths on a compliant surface. Moreover, Sainio et al have shown that the compressions performed on a mattress were significantly shallower [19]. Segal et al. indicate that such devices used on a dental chair may not be sufficiently effective due to the lack of seat stability [20]. Due to a different design of individual units, the current recommendation is to place a stool under a headrest in order to increase the stability of a chair, while maintaining high-quality CC. Some reports describe the higher amplitude of the chair's tilt during resuscitation without firstly supporting the headrest $(1.99 \mathrm{~cm})$, compared to using the support mentioned above $(0.43 \mathrm{~cm})[21,22]$.

There is a growing evidence that the most effective position for the rescuer performing CPR is a kneeling position, or using a dental stool in a case when resuscitation is carried out in a dental office [23-27]. The majority of units can be adjusted to the desired position, depending on the operator's preferences. An essential aspect is the experience and skills of a resuscitation team [28]. We must have in mind that there is usually a broad spectrum of employees in a dental office, including dentists, dental assistants, receptionists and dental hygienists.

A number of studies have proved differences in the quality of CPR depending on a type and level of education [29, 30]. The results of these reports differ depending on various factors. One of the studies pointed out that the quality of CPR was 


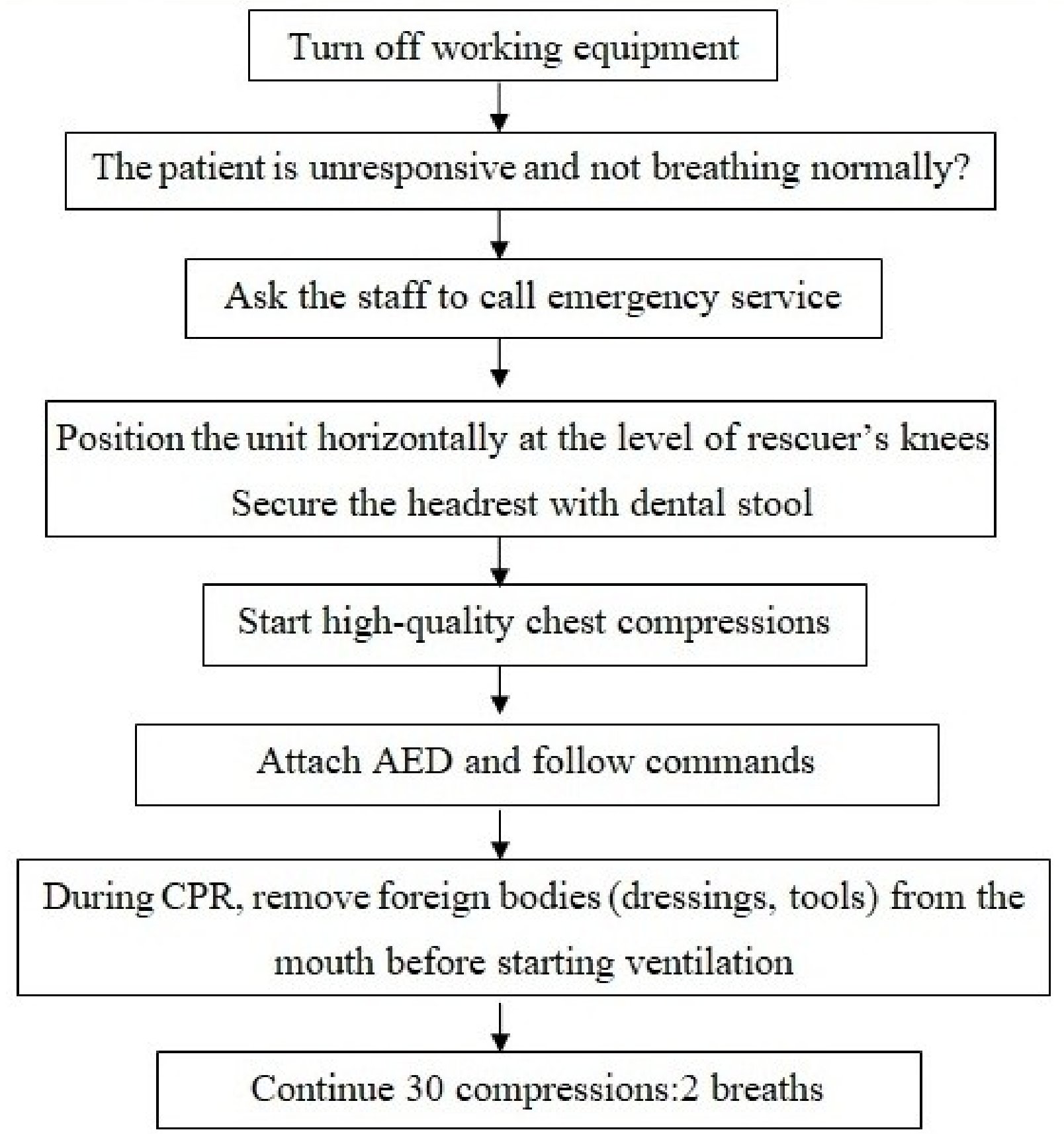

F I G U RE 3. Proposition of the resuscitation procedure.

higher among students than those actively working as nurses. It was also indicated that only $21 \%$ of respondents performed $\mathrm{CC}$ of at least $50 \%$ success rate. Better quality of $\mathrm{CC}$ was obtained by those who have received CPR training in the past six months [29]. Körber et al. showed a significant difference between the quality of $\mathrm{CC}$ between students and paramedics. The most appropriate rate of $\mathrm{CC}$ was also achieved in the group of paramedics. However, the study indicates the need to improve CPR skills in each of these groups [30].

In both studies mentioned above, particular attention was paid to the fact that for each group, the performed activities have not met the criteria of high-quality CPR. The need for constant education of medical staff and medical students in the field of CPR, as well as a more rigorous process of practical training, were also indicated.
In 2015 Malamed proposed the creation of resuscitation teams, which would include: a dentist, a dental assistant and a dental hygienist, and sometimes a nurse [31]. The leader of this team and a person directly responsible for patients' safety would be a dentist. The author also pointed to the fact that every member should undergo BLS training once a year. Such course should not only be organized in non-standard conditions, but also in a place of their daily work.

This form of training is known as "in situ simulation". It is based on creating conditions identical to reality, to faithfully reproduce the event and achieve the maximum realism of action. In situ simulation, in a valuable way, shows the level of knowledge and skills as well as gaps in work management [32]. We presented our proposition of the procedure of resuscitation in a dental office in Fig. 3. This procedure eliminates the 
provocation of secondary injuries. To optimize the ergonomics and effectiveness of the actions, we recommend that the unit should be positioned horizontally at the height of $45 \mathrm{~cm}$ (at the height of the knees of the rescuer's) as the most convenient position for providing CC. This arrangement of the unit ensures that the patient's chest is adjusted to the height of the rescuer.

\section{Conclusions}

It was proved that performing of $\mathrm{CC}$ on a dental chair with acceptable range of current quality parameters might be possible. Therefore, the procedure we presented might be essential in providing safe and efficient resuscitation in a dental office. Nevertheless, better technique and more precise studies are strongly needed.

\section{Limitations}

The study group consisted of individuals well-trained in CPR and acquainted with manikins. Therefore, it cannot wholly reproduce a group of people who may likely be the witnesses of SCA in a dental office.

The quality of chest compressions assessed by the simulator is based on three main factors: rate, depth and recoil. These $\mathrm{CC}$ quality parameters were precisely defined by the ERC. Achieving the correct values leads to the highest outcome. All quality parameters are interrelated and should be carefully interpreted. At the moment, there is no single ideal indicator of the quality of chest compressions that could be measured in simulation based research.

Our results showed that some of the $\mathrm{CC}$ were suboptimal (i.e. below $50 \mathrm{~mm}$ ) which is not effective in real life. Even though not significantly different statistically, it could be due to a small sample size. Performing real resuscitation is associated with high levels of stress that can also interfere with the performance. This was also not considered in our study. Moreover, the test lasted for 2 minutes. In daily practice, CPR is usually carried out much longer, and the quality of CC decreases over time.

Additionally, participants were aware of taking part in an experiment, what could have led to Hawthorne effect, meaning that they were trying to perform chest compressions at their very best. It could have also been developed because of researchers' presence. It has to be stressed out that the authors of the research refer to letters to editors because there are no sufficient original papers.

\section{ACKNOWLEDGEMENTS}

Thanks to all the peer reviewers and editors for their valuable opinions and suggestions.

\section{CONFLICT OF INTEREST}

The authors declare no competing interests.

\section{AUTHORS' CONTRIBUTIONS}

TK, RZ, MM designed the research study. MDa, MR, PR, BS, MD performed the research. RZ, ISA, TK, analyzed the data. TK, RZ, MP, MS, PR, BS wrote the manuscript. BC, BP, MP critically reviewed the manuscript and provided supervision. All authors contributed to editorial changes in the manuscript. All authors read and approved the final manuscript.

\section{ETHICS APPROVAL AND CONSENT TO PARTICIPATE}

The research protocol was approved by the Bioethics Committee of the Poznan University of Medical Sciences (No KB 69/20). Written consent to participate in the study was obtained from each participant.

\section{REFERENCES}

[1] Atherton GJ, McCaul JA, Williams SA. Medical emergencies: Medical emergencies in general dental practice in Great Britain. Part 1: their prevalence over a 10-year period. Br Dent J. 1999;186:72-79.

[2] Atherton GJ, McCaul JA, Williams SA. Medical emergencies in general dental practice in Great Britain, part III: perceptions of training and competence of GDPs in their management. Br Dent J. 1999;186 234-237.

[3] Arsati F, Montalli VA, Flório FM, et al. Brazilian Dentists' Attitudes About Medical Emergencies During Dental Treatment. J Dent Educ. 2010;74:661-666.

[4] Sooch S, Kaur A, Ahmed B. A ten year experience of medical emergencies at Birmingham Dental Hospital. BDJ. 2018;224:89-91.

[5] Girdler NM, Smith DG. Prevalence of emergency events in British dental practice and emergency management skills of British dentists. Resuscitation. 1999;41:159-67.

[6] Malamed SF. Medical emergencies in the dental surgery. J Ir Dent Assoc. 2016;6:302-308.

[7] Malamed SF. Medical emergencies in the dental surgery. Part 1: preparation of the office and basic management. J Ir Dent Assoc. 2015;61:302-308.

[8] Müller MP, Hänsel M, Stehr SN, et al. A state-wide survey of medical emergency management in dental practices: incidence of emergencies and training experience. Emerg Med J. 2008;25:296-300.

[9] Monsieurs KG, Nolan JP, Bossaert LL, et al. European Resuscitation Council Guidelines for Resuscitation 2015: Section 1. Executive Summary. Resuscitation. 2015;95:1-80.

[10] Wilson MH, McArdle NS, Fitzpatrick JJ, et al. Medical emergencies in dental practice. J Ir Dent Assoc. 2009;55:134-143.

[11] Qwen CP, Mizra N. Medical emergencies in dental practices in South Africa. S Afr Dent J. 2015;70:300-303.

[12] Laurent F, Segal N, Augustin P. Chest compression: Not as effective on dental chair as on the floor. Resuscitation. 2010;81:1729.

[13] Perkins GD, Handley AJ, Koster RW, et al. European Resuscitation Council Guidelines for Resuscitation 2015: Section 2. Adult basic life support and automated external defibrillation. Resuscitation. 2015;95:8199.

[14] Truhlář A, Deakin CD, Soar J, et al. European Resuscitation Council Guidelines for Resuscitation 2015: Section 4. Cardiac arrest in special circumstances. Resuscitation. 2015;95:148-201.

[15] Kemp K, Webb S, Jevon P. Moving and handling techniques in the dental chair. Dental Nursing. 2015;11:199-201.

[16] Lepere AJ, Finn J, Jacobs I. Efficacy of cardiopulmonary resuscitation performed in a dental chair. Aust Dent J. 2003;48:244-247.

[17] Takeshi Y, Kazu-ichi Y, Kunio S. Efficacy of external cardiac compression in a dental chair. Resuscitation . 2008;79:175-176.

[18] Field R, Soar J, Davies R, et al. The impact of chest compression rates on quality of chest compressions - A manikin study. Resuscitation. 2012;83:360-364. 
[19] Sainio M, Hellevuo $H$, Huhtala $H$, et al. Effect of mattress and bed frame deflection on real chest compression depth measured with two CPR sensors. Resuscitation. 2014;85:840-843.

[20] Segal N, Laurent F, Maman L, et al. Accuracy of a feedback device for cardiopulmonary resuscitation on a dental chair. Emerg. Med. J. 2012;29:890-893.

[21] Fujino H, Yokoyama T, Yoshida K, et al. Using a stool for stabilization of a dental chair when CPR is required. Resuscitation. 2010;81:502.

[22] Ito S, Karube N, Hirokawa J, et al. The Effect of the Use of a Stabilizer and Different Height Settings on the Stability of the Dental Chair when Performing High-Quality Chest Compressions. Emergency Medicine: Open Access. 2015;5:268-272.

[23] Yun SW, Lee BK, Jeung KW, et al. The effect of inclined step stool on the quality of chest compression during in-hospital cardiopulmonary resuscitation. Am J Emerg Med. 2014;32:851-855.

[24] Hong CK, Park SO, Jeong HH, et al. The Most Effective Rescuer's Position for Cardiopulmonary Resuscitation Provided to Patients on Beds: A Randomized, Controlled, Crossover Mannequin Study. The J Emerg Med. 2014;46:643-649.

[25] Oh J, Chee Y, Lim T, et al. Chest compression with kneeling posture in hospital cardiopulmonary resuscitation: A randomised crossover simulation study. Emerg Med Australas. 20014;26:585-590.

[26] Lewinsohn A, Sherren P, Wijayatilake D. The effects of bed height and time on the quality of chest compressions delivered during cardiopulmonary resuscitation: a randomised crossover simulation study. Emerg Med J. 2011;29:660-663.

[27] Oh J, Kim C, Kim S, et al. Comparison of chest compressions in the standing position beside a bed at knee level and the kneeling position: a non-randomised, single-blind, cross-over trial. Emerg Med J. 2013;31:533-535.

[28] Zideman DA, De Buck ED, Singletary EM, et al. European Resuscitation Council Guidelines for Resuscitation 2015 Section 9. First aid. Resuscitation. 2015;95:278-287.

[29] Nyman J, Sihvonen M. Cardiopulmonary resuscitation skills in nurses and nursing students. Resuscitation. 2000;47:179-84.

[30] Körber MI, Köhler T, Weiss V, et al. Quality of Basic Life Support - A Comparison between Medical Students and Paramedics. J. Clin. Diagn. Res. 2016;7:33-37.

[31] Malamed SF. Medical emergencies in the dental surgery. Part 1: Preparation of the office and basic management. J Ir Dent Assoc. 2015;61:302-308. Czekajlo M, Dabrowska A. In situ simulation of cardiac arrest. Disaster Emerg Med J. 2017;2:116-119.

[32] Czekajlo M, Dabrowska A. In situ simulation of cardiac arrest. Disaster Emerg Med J. 2017;2:116-119.

How to cite this article: Tomasz Kłosiewicz, Mateusz Puślecki, Radosław Zalewski, Michał Mandecki, Ilona Skitek-Adamczak, Maciej Sip, et al. Quality of Chest Compressions on A Dental Chair - A Randomized Simulation Trial as Observation in Support of A Procedure Proposal. Signa Vitae. 2020;16(2):175-181. doi:10.22514/sv.2020.16.0073. 